\section{Algunas visiones sobre la disciplina, práctica y concepto de la salud pública}

\section{RESUMEN}

Las definiciones de Salud Pública son múltiples, la OMS establece que son las acciones colectivas e individuales del estado y la sociedad civil, dirigidas a mejorar la salud de la población, pero otros autores han acentuado de manera distinta ciertos aspectos de acuerdo a su contexto politico y social. En el siguiente estudio se investigan algunas visiones sobre la salud pública en la Escuela de Salud Pública Dr. Salvador Allende de la Universidad de Chile, con una metodología cualitativa a través de entrevistas en profundidad a siete académicos. Se recogieron aportes de gran diversidad, muy influidos por sus vivencias. Destacaron la necesidad de entender la salud pública vinculada a la comunidad, comprometida con sus problemáticas y orientada hacia los cambios sociales. Señalan además los efectos de la dictadura y dejan ver problemáticas en torno a las consecuencias de la industria de los medicamentos, el mercado de la enfermedad, la influencia de éste sobre los contenidos de la investigación, las limitaciones para el trabajo interdisciplinario, carencias de la Escuela y la concordancia con la formación que se entrega en el pregrado y el discurso institucional.

Palabras Clave: Concepto de salud pública, definición de salud pública, salud pública en la Universidad de Chile, Escuela de Salud Pública.

\section{SOME VISIONS ON PUBLIC HEALTH, DISCIPLINE, PRACTICE AND CONCEPT}

\section{ABSTRACT}

There are multiple definitions of public health. The WHO established that public health refers to the collective and individual actions of governments and civil society, directed at improving population health. But other authors have highlighted different aspects according to their political and social context. In the following study, different visions of public health within the Dr. Salvador Allende School of Public Health of the University of Chile were investigated, using a qualitative methodology in which depth interviews were carried out with seven professors. A variety of responses were collected, each heavily influenced by the interviewee's experience. The need to understand public health as a disciple that is linked to the community, committed to understanding problems and oriented toward social change, were all highlighted. They also described the effects of the dictatorship and issues such as the pharmaceutical industry, the marketing of health and disease, the influence of these issues on research, the limitations of interdisciplinary work, the deficiencies of the School of Public Health, their approval of undergraduate and graduate training being offering, and the institutional discourse. 


\section{INTRODUCCIÓN}

La definición de Salud Pública considerada como clásica es la aportada por Winslow, que la concibió como "el arte y la ciencia de prevenir las enfermedades, recuperar la saludy evitar las consecuencias de la enfermedad mediante la acción colectiva de la población.". Por supuesto que han existido otras, pero varias de ellas en un intento de generar amplitud han devenido en imprecisión y ambigüedad ${ }^{2}$. La OMS sin embargo logró una notable precisión aclarando que "Salud Pública son las acciones colectivas e individuales, tanto del Estado como de la sociedad civil, dirigidas a mejorar la salud de la población"3. Esto fue importante porque sobrepasó las nociones de salud como un bien público con altas externalidades, precisando que las acciones colectivas son el grueso de las acciones en salud pública, por lo que ha preservado su relevancia hasta la actualidad.

Navarro advierte que la búsqueda de una definición no es sencilla ya que enfrenta dos grandes problemas, el primero en relación al termino "salud", que al ser entendido de manera biopsico-social obliga a incluir en el concepto intervenciones fuera del ámbito clínico incluyendo la producción, distribución de la renta, consumo, vivienda, trabajo, ambiente, etc. Y el segundo relativo a la delimitación de la "población", en la que no pueden incorporarse solo los sujetos enfermos sino que debe incluir también a los vulnerables ${ }^{4}$. No debe extrañar que exista tal diversidad en las definiciones de salud pública si a lo largo del tiempo estos límites se han ido modificando; en éste como en todos los campos del saber el significado que se confiere a cada término es producto de procesos sociales de significación y legitimación que dan cuenta de intereses predominantes de clases, grupos, actores y movimientos, evidenciando contenidos ideológicos, científicos y tecnológicos determinado por las condiciones históricas ${ }^{5}$. A juicio de Frenk en la historia del término salud pública los elementos que han sido más prominentes son: la acción gubernamental, el rol de la comunidad organizada, los servicios colectivos, y los servicios preventivos, los que siguen estando abiertos al debate en la actualidad ${ }^{6}$.

La "Nueva Salud Pública" una de las corrientes contemporáneas más influyentes de Latinoamérica en respuesta a la llamada "Crisis de la Salud Pública" coincidente con la instalación del neoliberalismo en los países dependientes, tiene entre sus exponente a Terris, quién buscó actualizar la definición de Winslow señalando que "La salud pública es la ciencia y el arte de prevenir las dolencias y las discapacidades, prolongar la vida, fomentar la salud (promoción) y la eficiencia física y mental, mediante esfuerzos organizados de la comunidad para sanear el medio ambiente, controlar las enfermedades infecciosas y no infecciosas, así como las lesiones, educar al individuo en los principios de la higiene personal, organizar los servicios para el diagnóstico y tratamiento de las enfermedades para la rehabilitación, así como desarrollar la estructura social que asegure a cada miembro de la comunidad un nivel de vida adecuado para el mantenimiento de la salud y el desarrollo de sus potencialidades." ". Éste y otros movimientos como el de la "Salud Colectiva" han intentado generar puntos de encuentro en las corrientes de renovación en el ámbito científico, práctico y profesional, situando el complejo promoción/salud/enfermedad/cuidado en una nueva perspectiva paradigmática a través de mayor participación de la sociedad ${ }^{8}$.

Montoya Aguilar en una revisión reciente trata de agrupar las definiciones que se han ido aportando al planteamiento original de Winslow, destacando a modo de conclusión que no corresponden a alternativas excluyentes, sino a expresiones que acentúan de manera diversa ciertos aspectos?. Aunque no se puede hablar de salud y en particular de salud pública sin apreciar la constante pugna entre corrientes filosóficas individualistas que privilegian el sujeto y las corrientes colectivistas más interesadas en la dimensión social, así como tradiciones positivistas y reduccionistas se enfrentan a los enfoques holísticos y dinámicos ${ }^{5}$.

La Escuela de Salud Pública fundada en 1943 en un esfuerzo conjunto de la Universidad de Chile, la Dirección General de Sanidad y el Instituto Bacteriológico, con el apoyo de la Fundación Rockefeller $^{10}$, en un contexto de expansión latinoamericana de una práctica médica concebida desde el positivismo ${ }^{8}$, fue un pilar en la construcción y consolidación del Sistema Nacional de Salud a mitad del siglo pasado; sufrió fuertes embates durante los años de dictadura, y hoy día enfrenta desafíos diversos ${ }^{10}$ por un lado con movimientos que pujan por superar las visiones reduccionistas y asumir la Salud Pública desde una perspectiva 
holística y compleja ${ }^{11}$, mientras a nivel internacional otros actores llaman a hacer de la gestión económica su principal campo de desempeño ${ }^{12}$.

¿Qué es hoy la salud pública dentro de la Escuela de Salud Pública de la Universidad de Chile? y ¿de dónde se nutre el concepto? son el motivo de éste estudio.

\section{MATERIALES Y MÉTODOS}

La presente investigación se desarrollo entre agosto y septiembre del 2012 en la Escuela de Salud Pública Dr. Salvador Allende de la Universidad de Chile, que fue la unidad de análisis de este trabajo.

La muestra fue definida como no probabilística por conveniencia. La elección de los entrevistados se hizo en conjunto con dos académicos de la escuela y se fue ampliando en la medida que se sucedían entrevistas y aparecían aspectos relevantes de tomar en consideración. Finalmente entre los criterios de selección de los entrevistados estuvo: el nivel académico, el considerar su visión como referente de opinión dentro de la escuela y el aporte de que podía hacer a la investigación desde la particularidad de su ámbito de desempeño. Se buscó representar a cada una de las divisiones, así como incluir a profesionales de distinta procedencia, formación y visión política, para aumentar la diversidad de discursos posibles.

Se entrevistó a siete académicos de acuerdo a los criterios mencionados, de ellos cuatro profesionales de la salud y tres de las ciencias sociales. Las divisiones quedaron representadas con tres académicos de Política y Gestión, uno de Epidemiología, uno de Bioestadística, y dos de Promoción de la Salud.

Se empleó un enfoque con características fenomenológico-etnográfico utilizando como herramienta entrevistas en profundidad y como instrumento un formulario semiestructurado con las preguntas: ¿Qué es la salud pública?, ¿Cual es su aporte?, ¿Cual fue su motivación?, ¿Su visión es de consenso? Se grabaron y transcribieron la totalidad de la entrevistas con consentimiento de los involucrados y fueron analizadas por dos investigadores de forma independiente.

Para el análisis de la información se utilizó la codificación abierta en el contexto del método de comparación constante, descrito para una primera etapa de análisis para la teoría fundada ${ }^{13}$. Este análisis también fue realizado en paralelo y en forma independiente por dos investigadores.

\section{RESULTADOS}

La historia de los sujetos y su anclaje en la Salud Pública.

Una académica cuya profesión de origen proviene de las ciencias sociales optó por un camino en la salud pública luego que en su trabajo se rechazara un informe acerca el aumento de la TBC asociado a la epidemia del SIDA. Ella se interesó en la aparición de esta enfermedad en los últimos años de la dictadura, teniendo ya alguna familiaridad con el tema a través de su padre médico.

Desde una acogedora oficina en el subterráneo, donde ofrece café de grano a sus visitas, relata: "me gustaba meterme por ejemplo en los datos y descubrir cual era el perfil de las guaguas que se estaban muriendo, eran los niños que venían o con problemas de deformación genética o con otros problemas por lo que fallecían al primer mes de vida." Realizó una tesis cualitativa trabajando con teatro popular educativo para prevenir las enfermedades respiratorias agudas en el sector del Barros Luco dado que "el drama en ese minuto era que mucho niños se morían en las casas, en los domicilios, por neumonía sin diagnóstico oportuno. "

Un episodio similar es relatado por otra académica que hizo su tesis sobre factores de riesgo de labio leporino. Sonriente detrás de un gran escritorio repleto de carpetas recuerda cómo recorrió las maternidades de Santiago en busca de datos sobre malformaciones sin saber que eso se llamaba epidemiología. Con posterioridad cuando ya había decidido formarse en salud pública, (dada una aversión a la clínica descubierta en el quinto año de la carrera), realizó un Magíster en Guatemala describiendo lo impactante de estar "en el lugar donde ocurrían las cosas" y el interés que sintió en "aprender de esa población e identificar aquellas cosas que realmente podían hacer un cambio". Este acercamiento a la comunidad mirado hoy desde un punto de vista etnográfico tuvo una profunda significancia en ella, "eso es lo que por lo menos a mí me hizo cambiar absolutamente, es decir: ni pienso en la bioquímica, ni en la nutrición, esta cuestión es lo fantástico, la gente." 
La clínica tampoco fue la vocación de un salubrista convertido a partir de las actividades desarrolladas en salud rural. Incorporándose a los equipos de trabajo de la Municipalidad descubrió el tema de la gestión: "empecé a descubrir este mundo súper interesante, había una racionalidad que se escapaba a la relación médico-paciente exclusiva, había otra lógica." Conoció a través de los programas la preocupación por el bajo control infantil, porque las poblaciones fueran vacunadas, porque se recibiera la alimentación complementaria, por el control del embarazo y el parto institucional garantizado. Así entró directamente a los temas de gestión y posteriormente a la salud pública a través de un programa de administración conjunto con la Escuela de Economía.

Desde la salud comunitaria, sin haber pasado aún por la clínica, llegó a la Salud Pública una profesional que se inició tempranamente en el mundo de las fundaciones y ONGs, "fue una preocupación desde los tiempos de la dictadura por las dificultades para el acceso a la salud como un derecho." Marcada desde un inicio por la relación entre la salud y las condiciones de vida reales de la población cuenta que: "hasta ese minuto sin una formación en salud pública, sino que era como el hacer y el aprender a través de la experiencia. Hasta que ahí viene la necesidad de sistematizar todo eso y de entenderlo de otra manera y hago mi formación en salud pública" Estuvo en África y España en varias ocasiones aportando a la construcción del modelo de sociedad cuyos valores compartía, trabajando en salud materno infantil, en el tema de violencia y desigualdad de género, en proyectos de salud familiar y salud sexual y reproductiva. "Me hizo mucho sentido lo cualitativo en salud pública (...) ayuda a entender por qué pasa lo que pasa"

Otro profesional de la salud entrevistado tuvo su primer contacto con la salud pública como ayudante en un estudio sobre embarazos adolescentes, se interesó en ella siendo dirigente estudiantil y se vinculó tempranamente a los grupos de salud a nivel poblacional, aprendió técnicas de educación popular y participó de la creación de una ONG. "A nivel de las poblaciones chilenas, particularmente de la Región Metropolitana, la organización de salud fue un foco de resistencia, eran mujeres pobladoras, que en torno al quehacer en salud lo que hacían era construir organiza- ción social, y yo colaboré en ese esfuerzo." Con la vuelta a la democracia se ofreció para colaborar en Conchalí, y terminó asumiendo varios cargos directivos, pese a que reconoce que ésta no es su verdadera vocación. Un fuerte interés en lo académico lo vinculó definitivamente a la escuela asumiendo importante responsabilidades por lo que reconoce como una deuda generacional, "éramos los que estábamos llamados a tratar de acortar la brecha entre las antiguas generaciones de salubristas y nosotros" dice en relación a quienes como él, se formaron en dictadura.

Otro académico proveniente de las ciencias sociales comenta en su ruidosa oficina compartida con colegas que su acercamiento vino después de haber colaborado en Venezuela en un centro latinoamericano de las Naciones Unidas, donde trabajó en la reforma del estado y en la formación de políticas sociales, "en ese momento era la punta en enfrentar toda la cosa esa de derecha, de decir que el mercado lo soluciona todo". A su juicio "puede ser solución de ciertas cosas pero no en la salud y no en la educación claramente.". $\mathrm{Su}$ rol inicial como salubrista estuvo abocado a desarrollar una mirada desde la gestión social o la gerencia social distinta a la gerencia puramente económica o financiera.

Finalmente un entrevistado que también se desempeña en un área no médica, relata que antes de egresar de su carrera en la Facultad de Economía se le presentó la oportunidad de hacer docencia, así llegó a la Facultad de Medicina involucrándose con la salud pública, aportando elementos de análisis cuantitativos a la investigación en salud, "el trabajo de estudiar situaciones de salud que le ocurren a los conjuntos de personas, eso me gusta, tener la posibilidad de diagnosticar y evaluar a través de los números". Su vinculación definitiva con la escuela es reciente y de eso da cuenta una oficina muy sobria, donde no abundan elementos decorativos ni personales.

\section{EL GOLPE Y SU IMPACTO EN LA ESCUELA}

En casi todas las entrevistas se menciona al Golpe Militar como un episodio relevante tanto en la historia personal como en el desarrollo de la Escuela de Salud Pública. Catalogado como un quiebre profundo que significó para destacados académicos el alejamiento, la muerte o el exilio. "Se perdió 
la continuidad de la escuela y no la ha recuperado tan fácil, esta cosa es muy difícil, fue un quiebre en el alma de la salud pública nacional".

Pese a la valoración positiva del importante desarrollo académico generado en los últimos años por la escuela, aun no ven que se alcancen los niveles de relevancia y repercusión nacional e internacional que la institución poseía antes de este evento. "Está en una etapa de renovación, esto no se puede considerar todavía ni te diría, si nos dijeran está a que nivel comparado con lo que era en los años, antes del golpe militar"

La Escuela de Salud Pública dejó de ser el centro generador de políticas del estado y pasó a convertirse en una institución cuya existencia resultaba "incómoda" para el gobierno militar. "Del punto de vista de la presencia de la Escuela de Salud Pública en el acontecer nacional, era un gobierno más bien adverso a los intereses públicos, por lo que significó una reducción de la presencia nuestra en el quehacer de la salud pública nacional".

Las reformas generadas durante la dictadura, desmantelaron en gran parte el sistema de salud que la Escuela había contribuido a consolidar e instaló principios ajenos al desarrollo del sistema público, con poco énfasis en lo promocional y lo colectivo, generando en la práctica un abandono. "Después del golpe de estado yo observé un deterioro del sistema público de salud, no había preocupación por invertir en el sistema público.”

\section{LA VISIÓN DE LA SALUD PÚBLICA EN CUANTO A OBJETIVOS Y ÁMBITOS DE ACCIÓN}

El primer elemento a destacar es que la salud pública es vista como una preocupación por la población, como la disciplina que se ocupa de evaluar, estudiar, coordinar y dirigir la salud de la población o los grandes colectivos del país. Se hace una clara diferencia con la medicina clínica que se hace cargo de los aspectos de la salud relacionados con lo individual. "Así como el médico clínico hace un diagnóstico de su paciente, el medico salubrista o el personal profesional de la salud pública debe ser capaz de hacer un diagnóstico y tratamiento de una población." El enfoque sobre su función está puesto en los grupos de personas, en la salud colectiva y no solo en pacientes o usuarios.
Se considera transversalmente como una herramienta para generar cambios, para identificar las claves del cambio y influir en la sociedad. El objeto de estos cambios es referido como: la búsqueda de una sociedad más equitativa con "un sistema que sea capaz de garantizar la salud poblacional con equidad", o como el impacto en política pública que puedan tener los resultados de investigaciones y proyectos "aportar desde el conocimiento que la salud pública permite levantar". La investigación genera evidencia para poner "de la mejor forma posible a disposición de los tomadores de decisiones" y así contribuir a generar transformaciones.

Se entiende como un ciencia aplicada cuyo quehacer es relevante en función de su capacidad de incidencia real o aplicación práctica, "la salud pública no se engolosina con el deleite intelectual de la generación de conocimiento" sino que "es un ciencia aplicada, que tiene una vocación de acción", que debe ser medida en términos de excelencia académica "no solo en el juicio de los pares, sino en la relación con la sociedad". En este sentido se destaca que la salud pública no permanezca indiferente de la realidad nacional y no se limite a manejarse en lo técnico de la profesión sino que se comprometa con las necesidades y los conflictos del país.

Que haya académicos dispuestos a hablar con la prensa, participar en discusiones, dar su opinión y mostrar resultados, se interpreta como que "la escuela de salud pública está comprometida con las necesidades y con los conflictos del país... Y a mí me parece muy bien, yo soy partidario de que las cosas sean así, digamos que uno no puede permanecer indiferente"

Así como se ve orientada a los cambios, la salud pública también es vista con una estrecha relación con lo político: "la actividad pública se mezcla con lo social cuando tiene que elaborar política de salud, y con lo político por la visión económica de estado y de desarrollo de país". Quienes la ejercen siempre son llamados a sostener una posición política, "una persona que está trabajando en salud pública no puede prescindir de tener una posición o una opinión respecto de si el estado va a tomar mayor o menor participación en el desarrollo de las política de salud pública; digamos por lo tanto que si creo que la salud pública está impregnada de lo político y social, no puede estar ajena." 
La neutralidad política de los salubristas es cuestionada desde varias miradas o calificada lisa y llanamente como inexistente. "cuando un salubrista trata de ponerse en el medio, ser ecuánime; no, esa cuestión no se puede, tu no puedes tratar de influir en una política pública siendo neutral, porque no existe la neutralidad". Se destaca la relevancia del posicionamiento político en la preocupación por el contexto social; "la gran mayoría de la gente de aquí primero se dicen progresistas, es como una primera aproximación y por lo tanto una gran preocupación por una realidad social que hay que transformar" Los profesionales deben "empujar el conocimiento que sea socialmente responsable y políticamente comprometido"; con distintos roles, "cada uno desde su perspectiva, a mi me gusta buscar las claves para el cambio (...) pero no hacer directamente los cambios".

Se agrega que un elemento consustancial en el quehacer de los salubristas: lograr una relación cercana entre la disciplina y las problemáticas sociales, centrando el foco fuera del sistema de atención de salud, no quedándose en la puerta mirando sino que involucrándose directamente con la realidad existente más allá de las instituciones y por sobre todo prestando atención a los determinantes sociales; "tú no puedes hacer salud ni salud pública si no estás involucrado, si no estás conociendo de alguna manera lo que las personas común y corrientes están viviendo, en materia de salud, en materia de calidad de vida, en todo lo que se relaciona con factores determinantes de la salud, especialmente en los determinantes sociales". En otras palabras "incorporando, participando con la comunidad y trabajando con la comunidad".

La aplicación de metodologías cualitativas a la salud pública otorgan la posibilidad de intervenir sobre los problemas sociales a la vez que se van investigando fenómenos humanos. Como ejemplo se coloca la metodología de investigación-acción que se desarrolla en la división de Promoción de Salud, a través de la cual se logra visibilizar temas que no aparecen fácilmente en las cifras.

Como área de desarrollo de la salud pública está la organización de la atención del sistema de salud que debe hacerse cargo no solo de donde están las enfermedades, cuáles son sus causas, cómo se ponen de manifiesto, cuál es la intensidad de su ocurrencia y cómo se distribuyen, sino también de la respuesta a estos problemas según su historia natural, "por un lado prevenir, evitar a lo mejor estas enfermedades y de qué manera enfrentarlas cuando se han puesto de manifiesto en sus distintas etapas".

Se menciona a la gestión como un instrumento necesario para optimizar el uso de recursos a partir de una realidad, donde los mercados de servicios médicos, medicamentos e insumos clínicos continuarán su boyante desarrollo, por tanto en la organización de la respuesta a los problemas de salud desde la salud pública se ve un espacio para gestionar y optimizar el uso de recursos de una industria que no se acaba, que son cada vez más caros, para que no represente una carga muy grande para la sociedad; "Yo siento que hay un desafío que es prístino, que es claro, que es congruente, si nos vamos a dedicar a esto, gestionemos bien, porque si gestionamos bien, va a alcanzar para más, va a alcanzar para todos, vamos a poder ser más justos con el tema, hay muy buenas razones para gestionar bien los servicios." En el aspecto de la salud pública relacionado con la gestión aparece mencionado un desafío en relación a la calidad, que aun no ha sido satisfecho por el GES al no incluirse entre sus garantías, "hay gente que le gusta ir al médico por un tema de estatus y hay enfermos que quieren asistencia y servicios de calidad, hay calidad que proveer".

Pese a opiniones que aun circunscriben a la salud pública como el área social por excelencia de la medicina, propia de "grandes médicos" que "optaban por la salud pública como un área de gran destaque", el grueso de las opiniones apuntan a considerarla como un espacio interdisciplinario, "yo no creo que la salud pública sea un problema de tipo médico o del ámbito de la salud, la veo como un espacio multidisciplinario", inclusive sosteniendo que más que interdisciplinario es transdisciplinario, de manera que la escuela podría estar perfectamente en la Facultad de Derecho o en la Facultad de Ciencias Sociales.

\section{LA RELACIÓN DE LA SALUD PÚBLICA CON EL MERCADO Y LOS PUNTOS DE DESENCUENTRO}

Aparece fuertemente plateado el problema del mercado y su relación con la salud pública en torno a lo cual podría decirse que existe un debate abierto 
sobre el que se identifican posiciones encontradas. "Alguna gente aquí (...), tienen una visión mucho más activista de las cosas, otros no, y probablemente los otros tienen una visión del sistema de salud pública chileno en que la parte que tiene que ver con negocios de la salud es muy importante; ninguno de ellos alaba eso, es solo que ahí está y es muy importante y tenemos que estar ahî".

Existe una visión crítica en lo relativo a la industria de las vacunas, el negocio de los laboratorios, medicamentos y al sistema de salud, también a negocio de la enfermedad y el énfasis asistencial de la red de atención. Hay preocupación por la penetración del mercado en todos los ámbitos de la salud y sus consecuencias en cuando a incentivos, que influyen por ejemplo el objeto de la investigación, que promueven la competencia entre los salubristas y vuelcan el sistema hacia el manejo de la enfermedad. "Acá hay un mercado de la enfermedad, y ese es el que mueve fuertemente los recursos y mueve todo, la formación médica, todo, cuyo propósito último es tratar enfermos, el gran tema del sector".

Hay una crítica abierta al positivismo como paradigma totalizante dentro de mundo biomédi$\mathrm{co}$, a la consideración casi exclusiva que en este ambiente adquieren las herramientas cuantitativas y a las mediciones ISI como valorización de la relevancia del trabajo académico, "somos de los pocos que estamos buscando desarrollar herramientas que no tengan que ver solo con ciencia positivista, con ese enfoque, y eso es súper difícil de meter en el mundo biomédico". Desde otra perspectiva: "porque justamente desde la mirada más social y desde la relevancia que tienen las circunstancias sociales en los comportamientos; finalmente en el acceso en el derecho a la salud lo cuantitativo se queda corto"

Pero existe también la visión de que la resistencia es inútil y los discursos pro-promoción son vacuos, "toda esa evidencia resulta ingenua al lado de la fuerza del mercado, el mercado arrastra con una fuerza increíble, por lo que se publican las cosas que están a favor y no las que están en contra, se las arregla el mercado para sostenerse a sí mismo en el tiempo sin cambios. En ese contexto se siente que el esfuerzo del discurso sanitario de promoción y prevención es ingenuo."

Otros debates al interior de la escuela en relación al ámbito de acción de la salud pública dicen relación con esta idea ya mencionada de volcar el trabajo hacia objetivos sociales en lugar de al mero desarrollo de la academia, "hay gente que quiere esto estrictamente como académico, y hay gente que está muy preocupada de si estamos respondiendo a las demandas reales o estamos haciendo juegos académicos, si estamos preocupados de los impactos que pueda tener, o de cómo cambiar esas políticas y como ayudar a cambiar las políticas o si simplemente estamos haciendo papers".

También chocan paradigmas a la hora de plantearse desde las metodologías cualitativas y enfocar el trabajo en la promoción de salud con la comunidad y no solo desde la atención. "La salud comunitaria se concibe hoy día como las acciones que desde esencialmente la atención primaria se hacen hacia la comunidad, y mucha gente lo que critica dice: eso es hacer salud sobre la comunidad no con la comunidad.". "Ahí hay una mirada, que es una mirada que a veces se ve como confrontacional, a veces en determinadas oportunidades con algo cuántico, lo que no es tal, a mi juicio son súper complementarias, y que tiene que ver también con una mirada como humanista también, de darle relevancia al saber y la experiencia de las personas como conocimiento científico".

Pese a la relevancia que se le otorga a que el cuerpo académico tome una posición y opine de las problemáticas nacionales, no todos los académicos comparten esta inclinación, "existen diferencias en cuanto a la necesidad de participación, no todos tienen la misma necesidad de participar, en solución de conflictos que no están en el ámbito inmediato de su quehacer".

El deseo de dotar de orientación social el objeto de la investigación dentro de la Escuela de Salud Pública parece chocar con que "los incentivos están puesto en las carreras individuales, que tan buen investigador demuestras tu ser, digamos, independientemente de lo que investigues finalmente". No existe un consenso a nivel de escuela que oriente la investigación hacia objetivos que no sean solo la suma de individualidades, "las líneas de investigación son al interior de la escuela, nunca se han fijado haciendo por ejemplo un gran trabajo de decir: miren ¿donde está la situación chilena, donde están los puntos de inflexión, donde están los puntos de continuidad, donde están los puntos de cambio?, ¿a que hay que apuntar si queremos tal y tal cosa?”. 


\section{DISCUSIÓN}

Los debates que cruzan la discusión sobre salud pública a nivel internacional tienen una expresión dentro de la escuela. El mencionado tema del mercado en salud, la industria de la enfermedad y la influencia de los consorcios económicos no es nuevo. Bustelo describió como en América Latina la economía aparece como una ciencia guía y sus recomendaciones constituyen el marco para la adopción de políticas sociales, "primero están las verdades macroeconómicas básicas y la política social se limita a recoger los heridos de las políticas económicas" ${ }^{24}$. Chile no queda al margen y da cuenta de una realidad en la que destacan las grandes diferencias sociales, con un sistema de salud que pareciera ahondar la privatización sin considerar sus impactos negativos, que en lugar de favorecer el abandono de la pobreza tienden a propiciarla ${ }^{15}$. Hay voces que se levantan para construir alternativas pese a los incentivos ya mencionados, en varias entrevistas se menciona la necesidad de consolidar grupos académicos capaces de generar una agenda propia, basada en la reflexión e identificación de problemáticas atingentes, pero el diagnóstico de la realidad pareciera ser aún un tanto pesimista.

Sobre la dinámica de trabajo dentro de la escuela pueden identificarse limitaciones a la integración y el trabajo interdisciplinario, pese a que todos los discursos recalcan su importancia y necesidad. Sin embargo la misma distribución física de la escuela sumada al carácter individual y competitivo de los proyectos de investigación y de los fondos, no lo favorecen. Las oportunidades que ofrece el cambio institucional, al que la escuela se ha abocado en el marco de la aplicación del Reglamento de Facultades y Unidades Académicas de la Universidad de Chile, son mencionadas con interés por varios académicos, como una posibilidad de reiniciar una dinámica integradora que dote el quehacer académico de las condiciones para desempeñarse bajo los paradigmas holístico y complejos que menciona Tarride ${ }^{11}$.

Para la escuela la docencia de pregrado es una responsabilidad ineludible y deseable, porque permite influir en la salud de la población a través de la formación de nuevos profesionales, constituyendo un semillero de futuros especialistas en salud pública ${ }^{16}$ pero vemos preocupantes contra- dicciones entre el deseo y la realidad. A juicio de los entrevistados la "formación que nosotros le damos a los médicos aquí en la facultad, es tan enfocada a lo clínico que cuesta mucho que salgan de esa mirada y lo vean desde esta perspectiva poblacional y social." Las facultades de medicina pueden ser ambientes perjudiciales para la formación médica ${ }^{2}$ y la evaluación que se hace es que el enfoque es predominantemente positivista en cuanto a los diseños epidemiológicos que se pueden realizar, mientras que a nivel internacional se discute sobre la crisis del modelo biomédico y la generalización de un nuevo paradigma que integre todas las características del bienestar humano ${ }^{17}$. En la Facultad de Medicina los estudiantes realizan trabajos donde analizan datos obtenidos de Internet para realizar diagnósticos de salud, sin acercarse directamente a la realidad de las localidades; algo tan destacado por los entrevistados y necesario para el conocimiento del contexto social que permita abordar las inequidades en sa$\operatorname{lud}^{18}$. No es de extrañar entonces que los alumnos llamen a la salud pública "salud pútrida", como se comenta en los pasillos, si el acercamiento a ella los enfrenta a modelos rígidos donde no se llevan a la práctica los elementos centrales del discurso.

No obstante se menciona la reforma curricular como otra gran oportunidad de realizar cambios en las mallas que otorguen a la formación de pregrado una congruencia con la idea de salud pública manejada por el grueso de la comunidad académica. Aunque se carecen aún de los campos clínicos adecuados para la territorialidad que exige el desarrollo de la medicina comunitaria.

A través de este estudio los profesionales de la Escuela dejan ver una serie de particularidades en la forma de concebir la Salud Pública con similitudes y diferencias marcadas por los distintos caminos recorridos antes de llegar a ella. La gama de matices percibidos da cuenta de la heterogeneidad de pensamiento dentro de esta casa de estudios, lo que constituye a todas luces una riqueza.

Desde mi personal perspectiva, la salud pública en la Escuela de Salud Pública de la Universidad de Chile es una disciplina que aborda la salud de los colectivos de personas, se encarga de evaluar y estudiar la situaciones de vida de estos, así como los determinantes sociales y otros elementos que configuran la salud en una dimensión holística, trabajando en estrecho vínculo con las comuni- 
dades y sus realidades, comprometiéndose con las necesidades de las personas, desde una mirada que trasciende el sistema de atención y adquiere un carácter interdisciplinario.

Como ciencia aplicada, su función no es solo el estudio sino la incidencia en la generación de cambios, con un importante componente político, que no puede estar ajeno al contexto en el cual se desenvuelve, ni permanecer al margen de sus problemáticas. Aborda la organización y gestión de la promoción, prevención y atención de la salud según su historia natural, optimizando su capacidad de respuesta a las necesidades y el uso de recursos. Se vale de herramientas cualitativas y cuantitativas, valorando las experiencias de los individuos y su visión de mundo como conocimiento científico. Pero estas distintas visiones sobre la salud pública poseen el dinamismo propio de los constructos humanos generados en base a la experiencia y están en continuo debate y confrontación entre la teoría y la práctica.

\section{BIBLIOGRAFÍA}

- Winslow. (1923). The significance of the modern Public Health Campaign. Yale: University Press.

- López, E. (2005). Hacia una nueva Salud Pública en Latinoamérica. Aten Primaria (Argentina), 336-8.

- OMS, O. y. (2003). La Salud Píblica en las Américas: Instrumento para a medición del desempeño para las funciones escenciales de la salud pública en el departamento de Caldas. Manizales: Centro Editorial Universidad de Caldas.

- Navarro, V. (1997). Concepto actual de la Salud Pública. En N. A. Martinez, Salud Pública (págs. 49-55). Madrid: MacGrawHill Interamericana.

- Gómez, R. (2002). La noción de Salud Pública: consecuencias de la polisemia. Rev. Fac. Nac. Salud Pública , 101-116.

- Frenk, J. (1994). La nueva Salud Pública. En Frenk, La salud de la población. Hacia una nueva Salud Pública. (pág. 76). Mexico DF: Fondo de Cultura Económica.
- Terris, M. (1992). Conceptos de Promoción de Salud: dualidades de la teoría de la Salud Pública. Washington: PAHO.

- Almeida Filho, N., \& Silva Paim, J. (1999). La crisis de la Salud Pública y el movimiento de la salud colectiva en Latinoamérica. Cuad. méd. soc. (Ros.). , 5-30.

- Montoya-Aguilar, C. (2006). ¿Qué se entiende, hoy, por Salud Pública? Cuad Méd Soc (Chile), 212-227.

- Universidad de Chile, E. d. (2003). Editorial: Nuestros Desafíos. Rev Chil Salud Pública, 117118.

- Tarride, M. (2005). Hacia la constitución de una Salud Pública compleja. Rev Chil Salud Pública , 169-174.

- Luis Sordo del Castillo, I. P. (2010). La Salud si tiene precio: papel de la Salud Pública en las evaluaciones economicas. . Gac Sanit, 431-432.

- Strauss, A.L. Y Corbin, J. (1990) Basics of Qualitative Research: Grounded Theory, procedures and techniques. Sage Publications. Newbury Park, CA.

- Bustelo. (2000). E. Salud y ciudadanía. Una mirada a la salud en el futuro. En La salud en América Latina. De la reforma para unos a la reforma para todos (págs. p. 253-95). Santiago de Chile: Editorial Sudamericana Chilena.

- Frenk. (2006). Bridging the divide: global lessons from evidence-based health policy in Mexico. The Lancet, 954 - 961.

- Solimano, \& Gonzalez. (2011). Construyendo la Salud Pública del Futuro. Planificación estratégica de la Escuela de Salud Pública de la Universidad de Chile. Calidad en la Educación, 293 - 303.

- Restrepo. (2001). Conceptos y definiciones. En Pomosión de la Salud. Como contruir una vida saludable. (págs. 24 - 33). Buenos Aires: Medica Panamericana.

- Diderichen, Evans, \& Whitehead. (2002). Bases sociales de las disparidades en Salud. En Desafío de la falta de equidad en Salud. De la etica a la acción. OPS - OMS. 


\section{Comentario: Una estrategia de sintaxis voluble}

Las personas solemos llenar de significado nuestro entorno aparentemente como una forma de construir un camino de suficiente certeza, cuando éste ya ha sido pavimentado empezamos a olvidar las espesuras y texturas colindantes y el final parece obvio. Preguntarse respecto de los significados de la salud pública en uno de los espacios de existencia cotidiana de los y las salubristas, me parece invita a problematizar respecto de certezas, matices y caminos posibles. Esto representa para nosotros una oportunidad de comprenderse en tanto colectivo y para el autor probablemente uno de los primeros lazos con el objeto de estudio. Por supuesto que los colores aparecen variopintos, combinados y por momentos de alto contraste, aparecen las historias de los peregrinos, su memoria y las heridas históricas extrañamente inesperadas. El autor del estudio tal vez se apresure en la construcción de una definición de salud pública ecléctica, un poco forzada en mi opinión, pero cuyo valor reside en recoger una postura personal luego del proceso de investigación.

Parafraseando, no existe una salud pública y ésta más bien aparece; en las aulas; en los centros de salud; en las oficinas oscuras y luminosas; en el conteo y registro constante; en las conversaciones del futuro y de lo no hecho. Le da sentido a nuestros pareceres, hace posible anhelos de justicia, de conocimiento, de sobrevivencia, de existencia, articula prestigios y poderes para los implicados, ya sea éste en el campo científico o en el de la práctica disciplinar. Desde una perspectiva histórica por otro lado, la salud pública en sus inicios puede comprenderse como parte de una estrategia biopolítica, un impulso de autoconstrucción de un nuevo cuerpo social, una medicina socializada enmarañada a la política social y al estado, que busca aprehender de la mano de la ciencia la parte que le corresponde del dispositivo de la población. Ahora bien la parte que le corresponde tampoco resulta simple de revelar, desde cierta perspectiva el objeto de estudio -la salud- parece nítido y aprehensible, desde otra complejo, históricamente determinado y políticamente encadenado.

Hoy la articulación de la estrategia se mueve con mayor libertad entre estado, mercado y sociedad civil, adquiriendo mayor centralidad o marginalidad dependiendo de los desequilibrios de fuerzas políticas al interior y exterior del campo: Dentro del campo por otra parte es posible desagregar sus significancias en relación con las diversas configuraciones de militancias políticas y epistemológicas.

No existe una salud pública, existe una estrategia de voluble sintaxis que ha delimitado un campo científico y práctico, en permanente creación y debate, pero en el cual nos distinguimos con cierta facilidad de lado a lado.

Álvaro Lefio C. 


\section{Comentario: Una primera salida a terreno}

Cuando en el zócalo Alvaro Lefio me presentó a Julio el año pasado como interno de Salud Pública, no se me ocurrió pedirle esta especie de consultoría que finalmente produjo.

Encerrados en la oficina, lo primero que hice fue conocer sus inquietudes intelectuales y descubrir algunas señas del camino que lo llevó a decidir estar un mes en nuestra Escuela. Le agradezco profundamente el par de horas que me regaló esa tarde y el aire semi-conspirativo con que concordamos la realización de este trabajo como contenido de su internado.

Su texto refleja algo de lo que Julio aspiraba a encontrar, más allá de la putridez señalada por sus compañeros. Y lo que ha encontrado tiene signos de un Chile mitad siglo XX y mitad siglo XXI, marcado por una profunda nostalgia y algunas oposiciones dibujadas con cierto dramatismo o pesimismo, expresión esta última que cito textual.

Habiendo venido y partido y regresado hace algunos años a esta Escuela, mi balance no se sitúa exactamente en ese medio camino entre una edad de oro y la producción anaerobia con que nos mientan los alumnos del pregrado.

Para ser justos con el pasado, habría no solo que llenarlo de nostalgia, sino redibujarlo con las formas autoritarias del Chile pretendidamente de- mocrático (el decreto 602 de participación en salud, por ejemplo), en los ordenamientos aristocratizantes del iluminismo sanitarista, en los puntos ciegos de la medicina social: las cárceles, los locos, las mujeres sin útero. Y contraponerlos a las dificultades del presente: el uso de una sociología extemporánea y emaciada, una abismante incomprensión etnográfica de lo económico y la debilidad antropológica de los estudios de los mercados en salud. Todo esto, bajo un mar de fondo de absoluta indiferencia a la producción intelectual procedente de los estudios de ciencia y tecnología (STS).

La pregunta ¿Qué es la salud pública? puesta en sus manos, intentaba ser como una brocha en las manos del aspirante a arqueólogo, con ocasión de su primera salida a terreno. Con ella pretendíamos encontrar algunos ejemplares en el ecosistema de definiciones. Y organizando esos hallazgos, reconocer especies, linajes y estrategias evolutivas.

Una vez adentrado nuestro consultor en el desafío de preguntar, premunido de unos términos de referencia más bien pobres, nos devuelve estos discursos como objetos y nos devuelve también sus preguntas y un animado autorretrato del salubrista cachorro.

Yuri Carvajal B. 